\title{
Glycogen storage disease due to aldolase A deficiency
}

INSERM

\section{Source}

INSERM. (1999). Orphanet: an online rare disease and orphan drug data base. Glycogen storage disease due to aldolase A deficiency. ORPHA:57

Glycogen storage disease due to aldolase A deficiency is an extremely rare glycogen storage disease (see this term) characterized by hemolytic anemia with or without myopathy or intellectual deficit. Myopathy can be severe enough to result in fatal rhabdomyolysis in some patients. A family with episodic rhabdomyolysis (triggerd by fever) without hemolytic anemia has recently been reported. 